\title{
Article \\ Influence of Spectral Filtration on Pulse Dynamics in Ring-Cavity Mamyshev Oscillator
}

\author{
Anastasia Bednyakova ${ }^{1,2, *(\mathbb{D})}$, Evgeny Kuprikov ${ }^{1}$, Irina Geraseva ${ }^{1}$ and Alexey Kokhanovskiy ${ }^{1}$ (D) \\ 1 Department of Laser Physics and Innovative Technologies, Novosibirsk State University, 1 Pirogova Str., \\ 630090 Novosibirsk, Russia; e.kuprikov@g.nsu.ru (E.K.); i.dubovaia@g.nsu.ru (I.G.) and kay@nsu.ru (A.K.) \\ 2 Institute of Computational Technologies SB RAS, 630090 Novosibirsk, Russia \\ * Correspondence: a.bedniakova7@g.nsu.ru
}

check for updates

Citation: Bednyakova, A.; Kuprikov,

E.; Geraseva, I.; Kokhanovskiy, A. Influence of Spectral Filtration on Pulse Dynamics in Ring-Cavity Mamyshev Oscillator. Appl. Sci. 2021, 11, 10398. https://doi.org/10.3390/ app112110398

Academic Editor: Boris Nyushkov

Received: 6 October 2021

Accepted: 2 November 2021

Published: 5 November 2021

Publisher's Note: MDPI stays neutral with regard to jurisdictional claims in published maps and institutional affiliations.

Copyright: (c) 2021 by the authors. Licensee MDPI, Basel, Switzerland. This article is an open access article distributed under the terms and conditions of the Creative Commons Attribution (CC BY) license (https:// creativecommons.org/licenses/by/ $4.0 /)$.

\begin{abstract}
Here we present a numerical study of pulsing build-up dynamics inside the fiber Mamyshev Oscillator (MO). The main scope of the investigation is to describe the influence of the spectral separation between the filters on self-starting $\mathrm{MO}$ dynamics and transition from multipulse to singlepulse generation regimes. It was found that Floquet stability analysis provides a straightforward way to determine whether the system will be self-starting or if it has to be excited by external source and predicts the repetition rate of the pulse train. We showed that spectrally overlapped bandpass filters provide reliable multi-pulse generation due to Faraday instability. Adiabatic increase in the spectral separation between the filters decreases the number of pulses down to single-pulse regime, therefore providing a flexible way to generate adjustable number of mode-locked pulses on demand.
\end{abstract}

Keywords: Mamyshev oscillator; spectral filtration; build-up dynamics; Faraday instability; Floquet stability analysis; numerical modeling

\section{Introduction}

Fiber Mamyshev oscillators (MO) attract a significant amount of attention from the laser community [1-6]. MOs combine advantages of fiber technologies, including compactness, efficiency,and excellent beam profile quality, and outstanding performance in terms of energy and recording spectral width of generated pulses. External compression of the pulses generated inside the MO cavity allows megawatt peak powers to be achieved [7].

One of the major issues of MO design is the self-starting of a pulsed regime. Since an $\mathrm{MO}$ cavity is spectrally closed to continuous waves, to start the pulsed regime, one has to excite sufficient optical power fluctuation. The first experimental demonstrations of the MO setup included a seed pulse source [8,9] as a mode-locking initiator. However, the need to use additional sources of ultrashort pulses significantly reduces the commercial appeal for such devices in real applications. Attempts to get rid of an external lasing source have resulted in the use of a starting arm inside the MO cavity. The starting arm commonly contains part of the mode-locking laser, for instance, a saturable absorber [10] or polarization-dependent elements [11]. The requirements for initial power fluctuations are not high; therefore, noisy Q-switched regime generated via the starting arm is sufficient to achieve mode-locking of MO cavity. Transition from a Q-switched to a mode-locked regime is provided by modification of the laser cavity, for example, via mechanical flipping of a mirror.

A recent approach to $\mathrm{MO}$ self-starting is based on spectral filters with overlapping bandwidths [12,13]. MO cavities with such filters have revealed reach dynamics of pulsing regimes, including soliton rains and harmonic mode locking [14]. At first sight, MOs containing only spectral filters have superior advantages over alternatives. However, in the work [10], the authors confirm that they did not achieve stable mode locking despite the fact that they had access to tuning the parameters of the spectral filters. In the work [15], the authors emphasize the need to occasionally mechanically shake the fiber cavity to 
achieve a stable mode-locking regime. Modulation of pumping power and application of a proper strategy to adjust the pumping power level and filter passband in linear MO cavity could solve the self-starting issue [16].

Here, we perform extensive research on the influence of spectral filtering on $\mathrm{MO}$ performance. It is known that dissipative Faraday instability may initiate multi-pulse generation inside fiber lasers in the case of zig-zag spectrally dependent losses [17-19]. Faraday instability may provide multi-pulse generation even for relatively low-power initial noise. We demonstrate that Floquet spectrum analysis is a powerful tool to examine self-starting possibility of the MO in this case. By analyzing the position and intensity of the maximum of the Floquet spectrum, it is possible to predict whether self-starting condition is satisfied and what the temporal separation will be between the mode-locked pulses at the laser output. We also studied transient dynamics from multi- to single-pulse generation, gradually increasing the spectral separation between the filters at the fixed pump power. The observed rich optical field dynamics, including hysteresis phenomena and formation of soliton molecules, makes the system attractive for further developing of new intelligent methods for active control of MO parameters.

\section{Numerical Model}

The simplified scheme of laser cavity, used in numerical simulations, is illustrated in Figure 1. The ring cavity includes 4 meter long PM SMF, two Yb-doped PM fibers $2.5 \mathrm{~m}$ long each, and two Gaussian spectral filters. The filters' reflectivities were equal to $R_{1}=60 \%$ and $R_{2}=10 \%$, while their bandwidth and central wavelength were tunable parameters. To model pulse propagation in PM fibers, we used generalized the Nonlinear Schrodinger Equation (NLSE) [20]:

$$
\frac{\partial A(z, t)}{\partial z}=-i \frac{\beta_{2}}{2} \frac{\partial^{2} A(z, t)}{\partial t^{2}}+i \gamma|A(z, t)|^{2} A(z, t)+\frac{g}{2} A(z, t),
$$

which includes saturated gain term $g(E)=g_{0} /\left(1+E / E_{\text {sat }}\right)$ if an active fiber is considered, where $g_{0}=25 \mathrm{~dB}$ is the small signal gain, $\mathrm{E}$ is the signal energy, and $E_{\text {sat }}=P_{\text {sat }} T_{R}$ is the saturation energy, $T_{R}$ is the cavity's round trip time. $A(z, t)$ is the slowly varying envelope associated with signal, $\beta_{2}=25 \mathrm{ps}^{2} / \mathrm{km}$ is the group velocity dispersion, and $\gamma=4 \mathrm{~W}^{-1} \mathrm{~km}^{-1}$ is the Kerr nonlinearity. Saturation energies corresponding to two gain fibers were $E_{\text {sat } 1}=10 \mathrm{~nJ}$ and $E_{\text {sat } 2}=30 \mathrm{~nJ}$. The gain profile was assumed to be Lorentzian with $40 \mathrm{~nm}$ gain bandwidth.

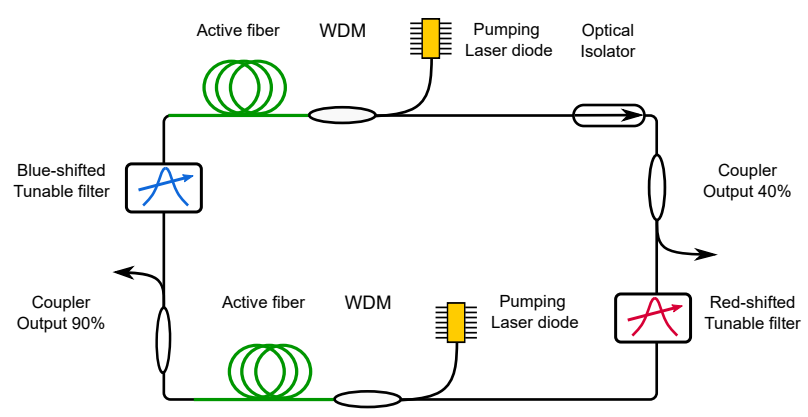

Figure 1. Simplified scheme of the Mamyshev oscillator used in numerical simulations.

\section{Analysis of Faraday Instability}

To find conditions for self-starting pulsed generation, we performed the linear Floquet stability analysis of stationary homogeneous solution and calculated spectrum of Faraday instability $[17,18,21]$. Two independent small perturbations were added to each spectral mode of a stationary solution and the optical field evolved during one cavity round trip. At the end of the round trip, a $2 \times 2$ matrix, indicating relative change of the real and imaginary parts of perturbation, was obtained. The matrix was numerically diagonalized, and the Floquet multipliers $F(\omega)$ were calculated as the eigenvalues of the matrix. If 
the modulus of the largest eigenvalue is less than one $F_{\max }(\omega)<1$, the amplitude of modulation does not grow. The average growth exponent for each mode can be calculated as $\ln \left(F_{\max }(\omega)\right)<0$, which corresponds to attenuation of the perturbation. In the opposite case, instability arises $\left(\ln \left(F_{\max }(\omega)\right)>0\right)$. Figure 2 shows the Floquet multipliers spectrum $F_{\max }(\omega)$, where a white line indicates a frequency, corresponding to the maximum growing rate of initial small signal modulation. This frequency defines the repetition rate of the pulses arising after stationary wave breaking.
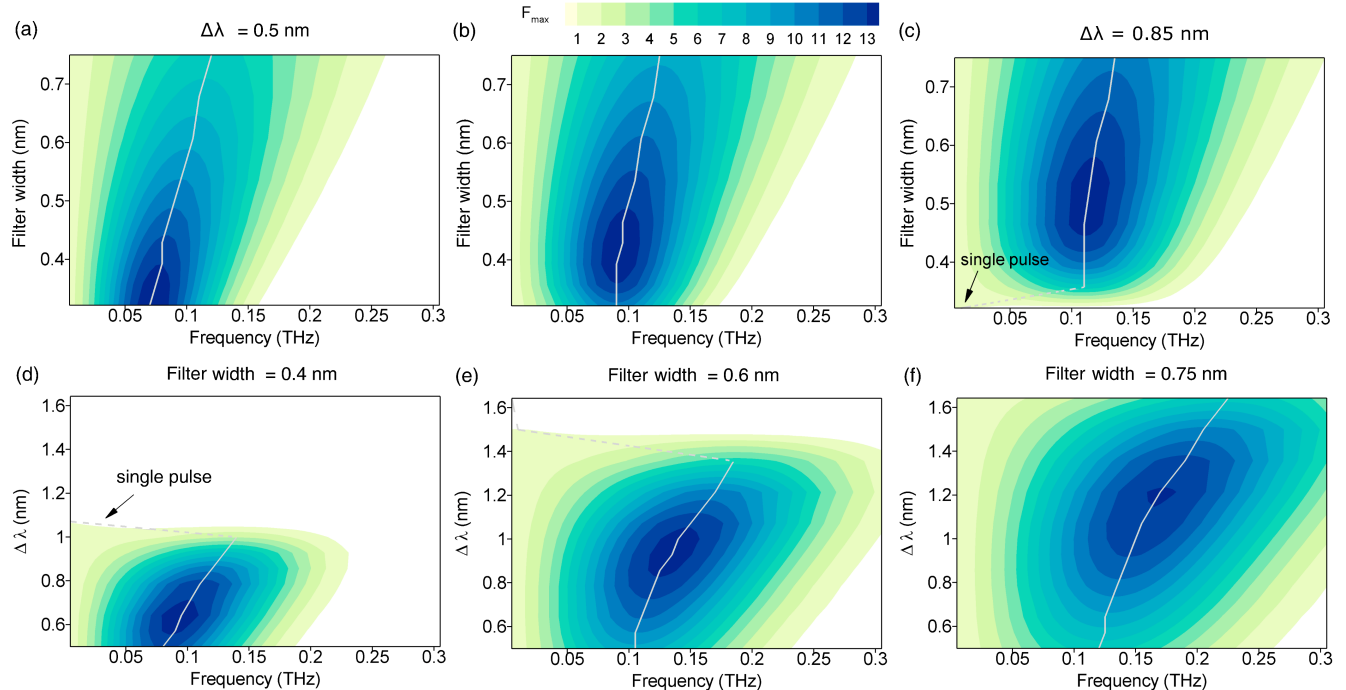

Figure 2. Faraday instability spectra $F_{\max }(\omega)$ for varying filters bandwidth (a-c) and spectral separation $(\mathbf{d}-\mathbf{f})$.

We then performed full NLSE-based numerical modeling of the Mamyshev oscillator, starting from "white" Gaussian noise at the first round trip, and compared the simulation results with theory predictions (Figure 3a). The results of the full modeling confirm that proper choice of filters bandwidth and spectral offset $\Delta \lambda$ between them (filters overlapping) leads to harmonic mode-locking of the fiber laser. Note that the repetition rate of the pulses is in good qualitative agreement with the theoretical predictions (Figure 3a). Figure $3 b$ illustrates how Faraday instability occurs during the first 50 cavity round trips. The green line depicts the number of pulses at the laser output, averaged over 100 realizations of the initial noise. At the first stage (from 1 to 12 round trip), the number of pulses in the temporal numerical window decreases and the optical field tends toward a continuous wave. Then, at the beginning of the second stage, the continuous wave becomes unstable and breaks into a strain of the pulses. The typical shape of the generated pulse train is shown in Figure 3c. If we continue to increase the spectral offset between the filters so that they no longer overlap, formation of a single pulse may occur (Figure 3d). The shape of a single pulse is close to parabolic, indicating similariton formation and evolution inside the laser cavity. Filter parameters, corresponding to a single pulse generation, are shown by black arrows in Figure 2 . The instability gain equals zero $\left(F_{\max }=1\right)$, because the filters does not overlap and continuous wave generation is suppressed. 

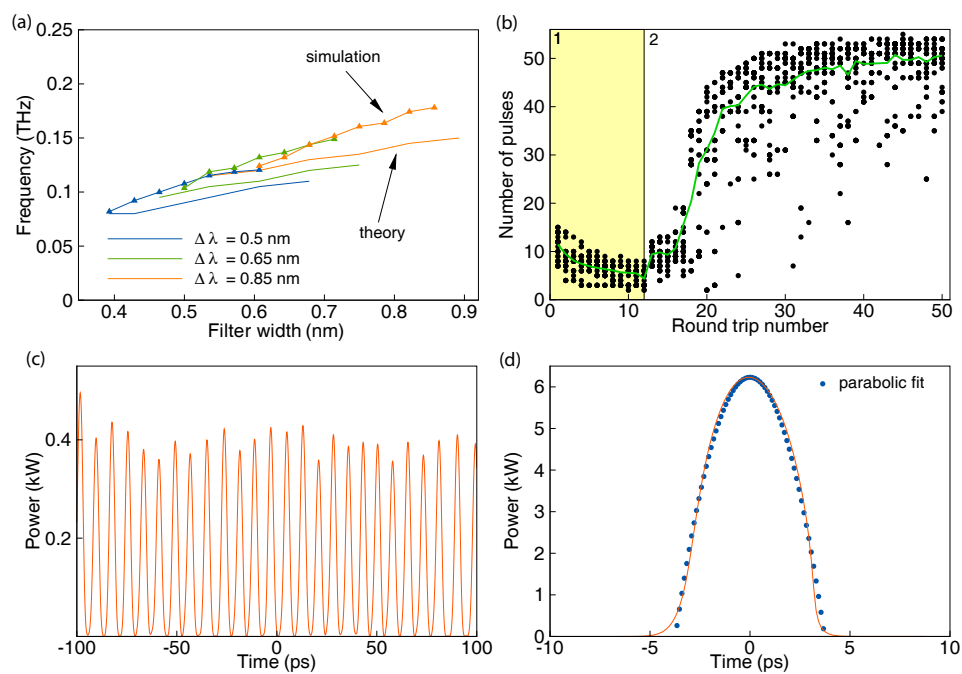

Figure 3. (a) The repetition rate of the output pulse train predicted in Floquet stability analysis and calculated using the full model. (b) Evolution of the pulse numbers in simulations during the first 50 round trips. Filter width $=0.6 \mathrm{~nm}, \Delta \lambda=0.7 \mathrm{~nm}$. $(\mathbf{c}, \mathbf{d})$ Characteristic temporal shape of the output field in the harmonic mode-locking and single-pulse regimes.

Therefore, if parameters of the tunable filters (bandwidth and spectral separation) are chosen in such a way that Faraday instability occurs (Figure 2), self-start of pulse train generation is initiated. Further transition from pulse train to a single pulse and conditions of successful transition are studied in the next section.

\section{Transition from Harmonic Mode-Locking to a Single Pulse Generation}

An interesting and practically important question remains, namely the dynamics of transition from harmonic mode-locking to a single-pulse generation, as well as the ability to control it. In this section, we investigate these dynamics resulting from the gradual increase in the spectral separation $\Delta \lambda$ between the filters. The filter width was fixed and equal to $0.6 \mathrm{~nm}$, while their central wavelengths symmetrically changed as a step-wise function. Note that filter parameters in the first step should satisfy conditions of Faraday instability discussed above. Figure 4 demonstrates how initial "white" Gaussian noise evolves to a single pulse when spectral separation between the filters varies from 0.7 to $2.8 \mathrm{~nm}$. After each step of $\Delta \lambda$, the intracavity field evolved during 50 roundtrips till the next step. The resulting temporal distribution of the output laser field measured at each cavity roundtrip is shown in the figure. We also observed that transient evolution of the optical field from harmonic mode-locking to single-pulse generation is accompanied by formation of soliton molecules with complex self-organization-soliton pairs, triplets, and $2+1$ molecules.

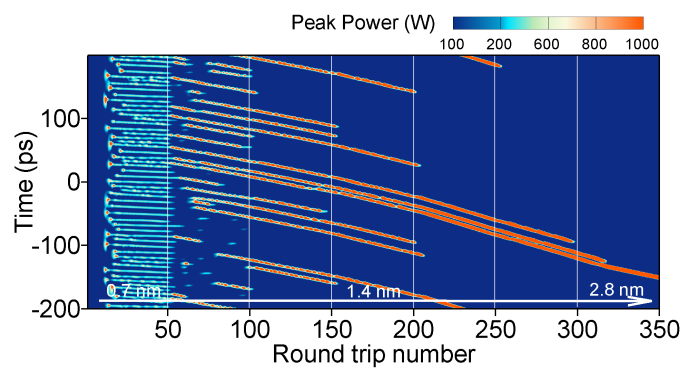

Figure 4. Evolution of the output field corresponding to varying spectral separation between the filters from $0.7 \mathrm{~nm}$ to $2.8 \mathrm{~nm}$. Filters width $=0.6 \mathrm{~nm}$. 
Figure 5 illustrates how a form of the initial field and the way of tuning the filters' spectral separation can affect the resulting output field. If a seed pulse is used at the first round trip, filter separation $\Delta \lambda=1.43 \mathrm{~nm}$ leads to stable generation of a single pulse (Figure 5a). Starting from the noise with $\Delta \lambda=1.43$ does not not lead to any modelocked regime (Figure $5 b$ ). Self-starting of mode-locking can be initiated if the initial spectral separation satisfies the condition of Faraday instability $(\Delta \lambda=0.7 \mathrm{~nm}$, Figure $5 \mathrm{c}-\mathrm{e})$. However, single pulse generation is not reached at $\Delta \lambda=1.43 \mathrm{~nm}$ anymore. Variation of the frequency step with which the wavelengths of the gratings move away from each other changes the pulse dynamics and leads to hysteresis. The same $\Delta \lambda=1.43 \mathrm{~nm}$ corresponds to a single multi-pulse generation or even the absence of mode-locked state.

(a)

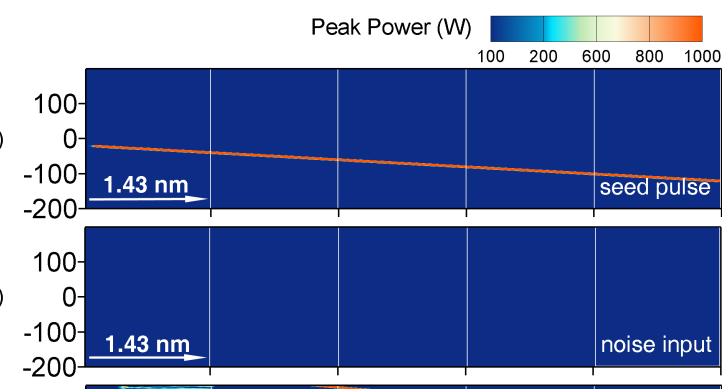

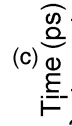

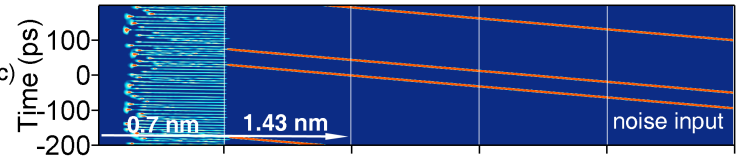

(d)

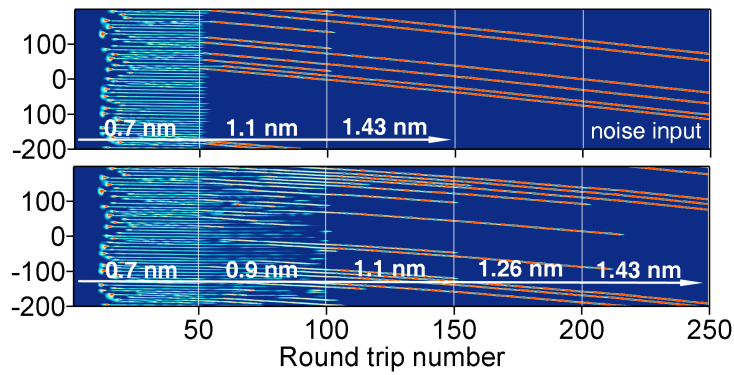

Figure 5. Evolution of the output field (a) from a seed pulse for $\Delta \lambda=1.43 \mathrm{~nm}$, (b) from noise for $\Delta \lambda=1.43 \mathrm{~nm}$, and (c-e) from noise for $\Delta \lambda$ changing from 0.7 to $1.43 \mathrm{~nm}$ with different spectral steps.

For a more complete picture of the output field dynamics, we performed numerical simulations of the Mamyshev oscillator, starting from 100 different realizations of initial "white" noise and $\Delta \lambda=0.7 \mathrm{~nm}$. After 50 roundtrips of the field propagation, the spectral separation $\Delta \lambda$ was chosen randomly in a given interval from 1.3 to $4 \mathrm{~nm}$, outside the Faraday instability region, and simulations continued from the current field during the next 50 roundtrips. If the field energy converged to zero, the process started again from the noise and $\Delta \lambda=0.7 \mathrm{~nm}$. The total number of steps along $\Delta \lambda$ did not exceed 5 . By changing the filter separation as described above, more than 27,000 lasing regimes were collected, including unstable noisy regimes. The variety of the obtained single- and multipulse regimes in the plane of $\Delta \lambda$ and output field energy is shown in Figure 6. Single pulse generation can be achieved in one step of $\Delta \lambda$, for spectral separation close to $2 \mathrm{~nm}$, or in several steps for $\Delta \lambda \geq 3 \mathrm{~nm}$ (Figure 6a). Dark blue circles depict the stable pulse generation with relative energy fluctuations less that $2 \%$ over the last 10 roundtrips, while medium blue circles correspond to transient regimes with pulse energy oscillating between two values. The pulse energy does not grow with the increase in spectral separation, because pump power did not change during simulations. It is noteworthy that there is a gap for $\Delta \lambda$ between 2 and $3 \mathrm{~nm}$, where single pulse generation was not observed (light blue circles in Figure 6a, noise-like regimes). Only multi-pulse generation is possible in this area. Figure $6 \mathrm{~b}$ shows the obtained multipulse regimes with the number of pulses from 2 to 4 . Dark-colored circles depict regimes with less than $2 \%$ energy fluctuations. The main trend 
is that smaller spectral separation leads to more pulses at the laser output. However, the same $\Delta \lambda$ could lead to formation of different number of pulses, depending on the way the filters are tuned. For example, for $\Delta \lambda=2 \mathrm{~nm}$, generation of a single pulse, soliton pair, triplet, or even quartet can be obtained.
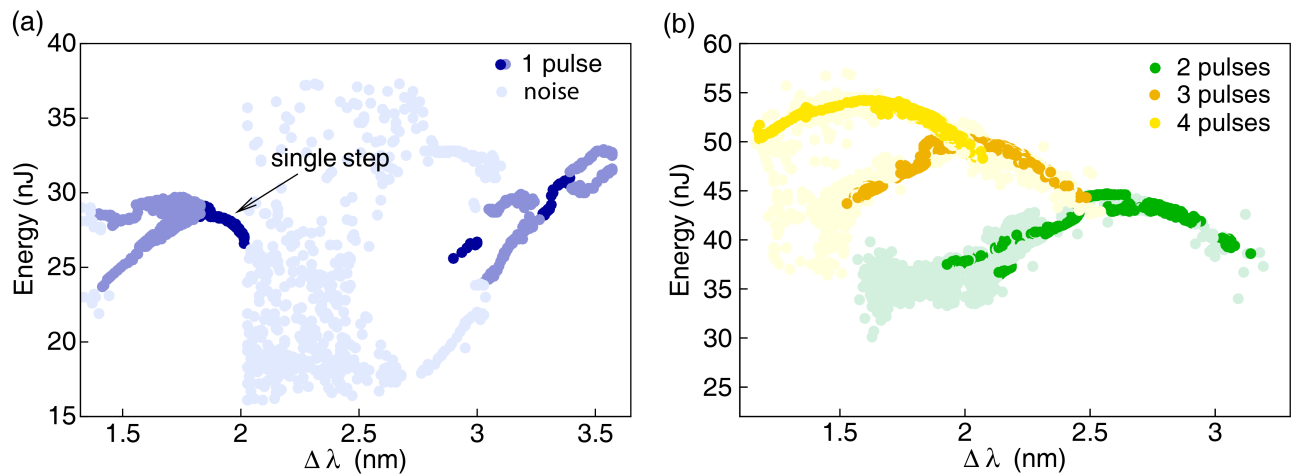

Figure 6. Dependence of the output field energy on the spectral separation between the filters, corresponding to a single (a) and multipulse (b) generation. Various possibilities of spectral tuning were considered, starting from $\Delta \lambda=0.7 \mathrm{~nm}$.

\section{Conclusions}

To conclude, we performed a detailed investigation of influence of spectral filtration on the output field dynamics of the ring-cavity MO. Using Floquet stability analysis, we examined self-starting possibilities of the $\mathrm{MO}$ and predicted repetition rates of the pulse trains generated due to Faraday instability. The results of the full NLSE-based numerical simulations are in good qualitative agreement with the theoretical predictions. We demonstrated that a gradual increase of the spectral filters separation with varying steps leads to rich transient dynamics between multi- and single-pulse generation regimes, including hysteresis phenomena and formation of soliton molecules. In further research, pump power should also be considered as a tunable parameter, leading to even more complicated dynamics. For this reason, it is important to develop intelligent methods for controlled tuning of the MO parameters to find the mode-locked regimes with required characteristics.

Author Contributions: Conceptualization, A.B. and A.K.; methodology, A.B.; software, A.B., E.K. and I.G.; validation, A.B., E.K. and A.K.; investigation, I.G., E.K. and A.B.; writing-original draft preparation, A.B. and A.K.; visualization, A.B. All authors have read and agreed to the published version of the manuscript.

Funding: This research was funded by the Ministry of Science and Higher Education of the Russian Federation (agreement No. 075-15-2021-1009).

Institutional Review Board Statement: Not applicable.

Informed Consent Statement: Not applicable.

Data Availability Statement: Not applicable.

Conflicts of Interest: The authors declare no conflict of interest.

\section{References}

1. Mamyshev, P. All-optical data regeneration based on self-phase modulation effect. In Proceedings of the 24th European Conference on Optical Communication, ECOC'98 (IEEE Cat. No. 98TH8398), Madrid, Spain, 20-24 September 1998; Volume 1, pp. 475-476.

2. Pitois, S.; Finot, C.; Provost, L.; Richardson, D.J. Generation of localized pulses from incoherent wave in optical fiber lines made of concatenated Mamyshev regenerators. J. Opt. Soc. Am. B 2008, 25, 1537-1547. [CrossRef]

3. Fu, W.; Wright, L.G.; Wise, F.W. High-power femtosecond pulses without a modelocked laser. Optica 2017, 4, 831-834. [CrossRef] [PubMed] 
4. Perego, A.M. High-repetition-rate, multi-pulse all-normal-dispersion fiber laser. Opt. Lett. 2017, 42, 3574-3577. [CrossRef] [PubMed]

5. Repgen, P.; Wandt, D.; Wienke, A.; Morgner, U.; Neumann, J.; Kracht, D. Yb-doped fiber Mamyshev oscillator with a few-mode gain fiber. In Fiber Lasers XVIII: Technology and Systems; Zervas, M.N., Ed.; International Society for Optics and Photonics, SPIE: Bellingham, WA, USA, 2021; Volume 11665. [CrossRef]

6. Nie, M.; Wang, J.; Huang, S.W. Solid-state Mamyshev oscillator. Photon. Res. 2019, 7, 1175-1181. [CrossRef]

7. Liu, Z.; Ziegler, Z.M.; Wright, L.G.; Wise, F.W. Megawatt peak power from a Mamyshev oscillator. Optica $2017,4,649-654$. [PubMed]

8. $\quad$ Regelskis, K.; Želudevičius, J.; Viskontas, K.; Račiukaitis, G. Ytterbium-doped fiber ultrashort pulse generator based on self-phase modulation and alternating spectral filtering. Opt. Lett. 2015, 40, 5255-5258. [CrossRef] [PubMed]

9. Poeydebat, E.; Scol, F.; Vanvincq, O.; Bouwmans, G.; Hugonnot, E. All-fiber Mamyshev oscillator with high average power and harmonic mode-locking. Opt. Lett. 2020, 45, 1395-1398. [CrossRef] [PubMed]

10. Boulanger, V.; Olivier, M.; Guilbert-Savary, F.; Trépanier, F.; Bernier, M.; Piché, M. All-fiber Mamyshev oscillator enabled by chirped fiber Bragg gratings. Opt. Lett. 2020, 45, 3317-3320. [CrossRef] [PubMed]

11. Sidorenko, P.; Fu, W.; Wright, L.G.; Olivier, M.; Wise, F.W. Self-seeded, multi-megawatt, Mamyshev oscillator. Opt. Lett. 2018, 43, 2672-2675. [CrossRef] [PubMed]

12. Samartsev, I.; Bordenyuk, A.; Gapontsev, V. Environmentally stable seed source for high power ultrafast laser. In Components and Packaging for Laser Systems III; International Society for Optics and Photonics: Bellingham, WA, USA, 2017; Volume 10085, p. 100850 S.

13. Želudevičius, J.; Mickus, M.; Regelskis, K. Investigation of different configurations and operation regimes of fiber pulse generators based on nonlinear spectral re-shaping. Opt. Express 2018, 26, 27247-27264. [PubMed]

14. Wang, P.; Yao, S.; Grelu, P.; Xiao, X.; Yang, C. Pattern formation in 2- $\mu \mathrm{m}$ Tm Mamyshev oscillators associated with the dissipative Faraday instability. Photonics Res. 2019, 7, 1287-1295. [CrossRef]

15. Luo, X.; Tuan, T.H.; Saini, T.S.; Nguyen, H.P.T.; Suzuki, T.; Ohishi, Y. All-Fiber Mode-Locked Laser Based on Mamyshev Mechanism With High-Energy Pulse Generation at 1550 nm. J. Lightwave Technol. 2020, 38, 1468-1473. [CrossRef]

16. Chen, Y.H.; Sidorenko, P.; Thorne, R.; Wise, F. Starting dynamics of a linear-cavity femtosecond Mamyshev oscillator. JOSA $B$ 2021, 38, 743-748. [CrossRef] [PubMed]

17. Staliunas, K.; Hang, C.; Konotop, V.V. Parametric patterns in optical fiber ring nonlinear resonators. Phys. Rev. A 2013, 88, 023846. [CrossRef]

18. Tarasov, N.; Perego, A.M.; Churkin, D.V.; Staliunas, K.; Turitsyn, S.K. Mode-locking via dissipative Faraday instability. Nat. Commun. 2016, 7, 12441. [PubMed]

19. Perego, A.M.; Smirnov, S.V.; Staliunas, K.; Churkin, D.V.; Wabnitz, S. Self-Induced Faraday Instability Laser. Phys. Rev. Lett. 2018, 120, 213902. [CrossRef] [PubMed]

20. Agrawal, G.P. Nonlinear Fiber Optics, 4th ed.; Elsevier: Amsterdam, The Netherlands, 2006.

21. Perego, A.M.; Tarasov, N.; Churkin, D.V.; Turitsyn, S.K.; Staliunas, K. Pattern Generation by Dissipative Parametric Instability. Phys. Rev. Lett. 2016, 116, 028701. [CrossRef] [PubMed] 